\title{
Bisphosphonate-induced Atypical Femoral Shaft Fracture
}

\author{
Muhammad Usman Baig ${ }^{1}$, Awais Maqbool ${ }^{2}$ \\ 1. Internal Medicine, Bahawal Victoria Hospital, Quaid-E-Azam Medical College, Bahawalpur, PAK 2. Multan Medical \\ College, Multan, PAK
}

Corresponding author: Muhammad Usman Baig, baigmd151@gmail.com

\begin{abstract}
Bisphosphonates are used to treat postmenopausal and glucocorticoid-induced osteoporosis, Paget's disease of bone, and malignant hypercalcemia. They have been related to atypical femur fractures. We describe an 83-year-old woman on long-term bisphosphonates presenting with an atypical femur fracture. She underwent a surgical procedure and, a few months later, she presented with a nonunion of the periprosthetic femoral fracture. She underwent an additional surgical procedure and discontinued bisphosphonate treatment. As more atypical femur fractures associated with bisphosphonate use are being reported in the literature, the risk of an atypical femur fracture with bisphosphonate use should not be ignored.
\end{abstract}

Categories: Orthopedics, Rheumatology

Keywords: bisphosphonates

\section{Introduction}

Osteoporosis is an important health challenge, and bisphosphonates provide the most benefit at the least cost. Structurally, bisphosphonates are analogues of pyrophosphate, with the feature of being resistant to enzyme or chemical breakdown. The bisphosphonates act by inhibiting the resorption of bone by osteoclasts; as a result, inhibiting bone mineralization and decreasing bone turnover.

The medical literature reports that bisphosphonates are a potential cause of atypical femur fractures. Atypical fractures are characterized by transverse and noncomminuted fracture patterns in the subtrochanteric or femoral shaft regions with a medial cortical spike at the fracture area. Other features include prodromal pain and a generalized thickening of the femoral cortices on radiographs [1-2]. We observed most of these features in the present case.

Received 09/13/2017 Review began 10/02/2017 Review ended 10/03/2017 Published 10/05/2017

(c) Copyright 2017

Baig et al. This is an open access article distributed under the terms of the Creative Commons Attribution License CC-BY 3.0., which permits unrestricted use, distribution, and reproduction in any medium, provided the original author and source are credited.

\section{Case Presentation}

An 83-year-old woman presented to our hospital with low-energy trauma resulting in pain in the right thigh, causing an inability to bear weight. She has a history of chronic obstructive pulmonary disease, type 2 diabetes mellitus, gastroesophageal reflux disease, and osteoporosis. She had an intertrochanteric fracture in the right neck of her femur in 2010, which was internally fixed using a dynamic hip screw (DHS) with a two-hole plate (Figure 1, Figure 2). 


\section{Cureus}

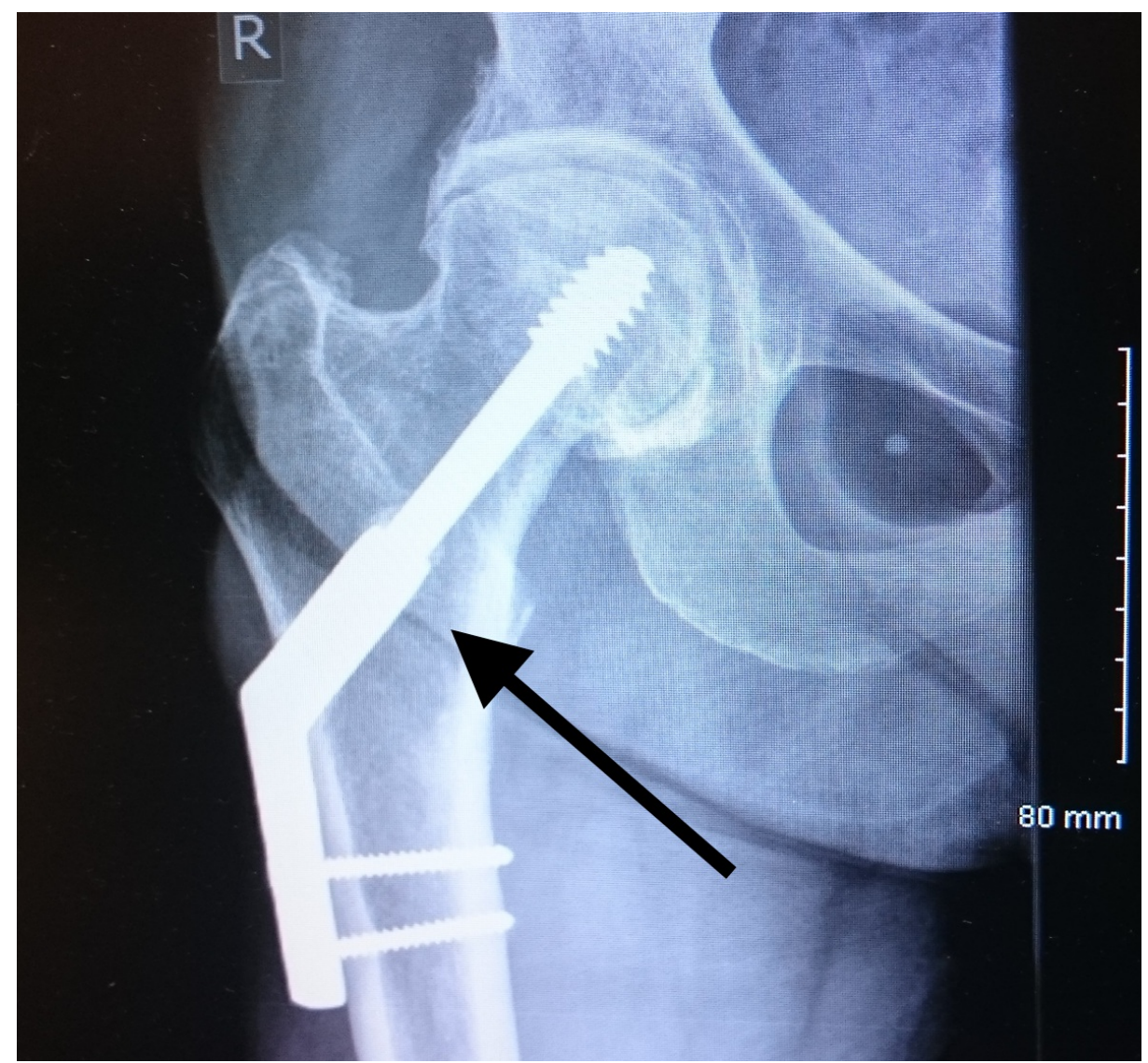

FIGURE 1: Dynamic hip screw with a two-hole plate for the neck of the femur fracture

Arrow showing the two-hole dynamic hip screw and plate

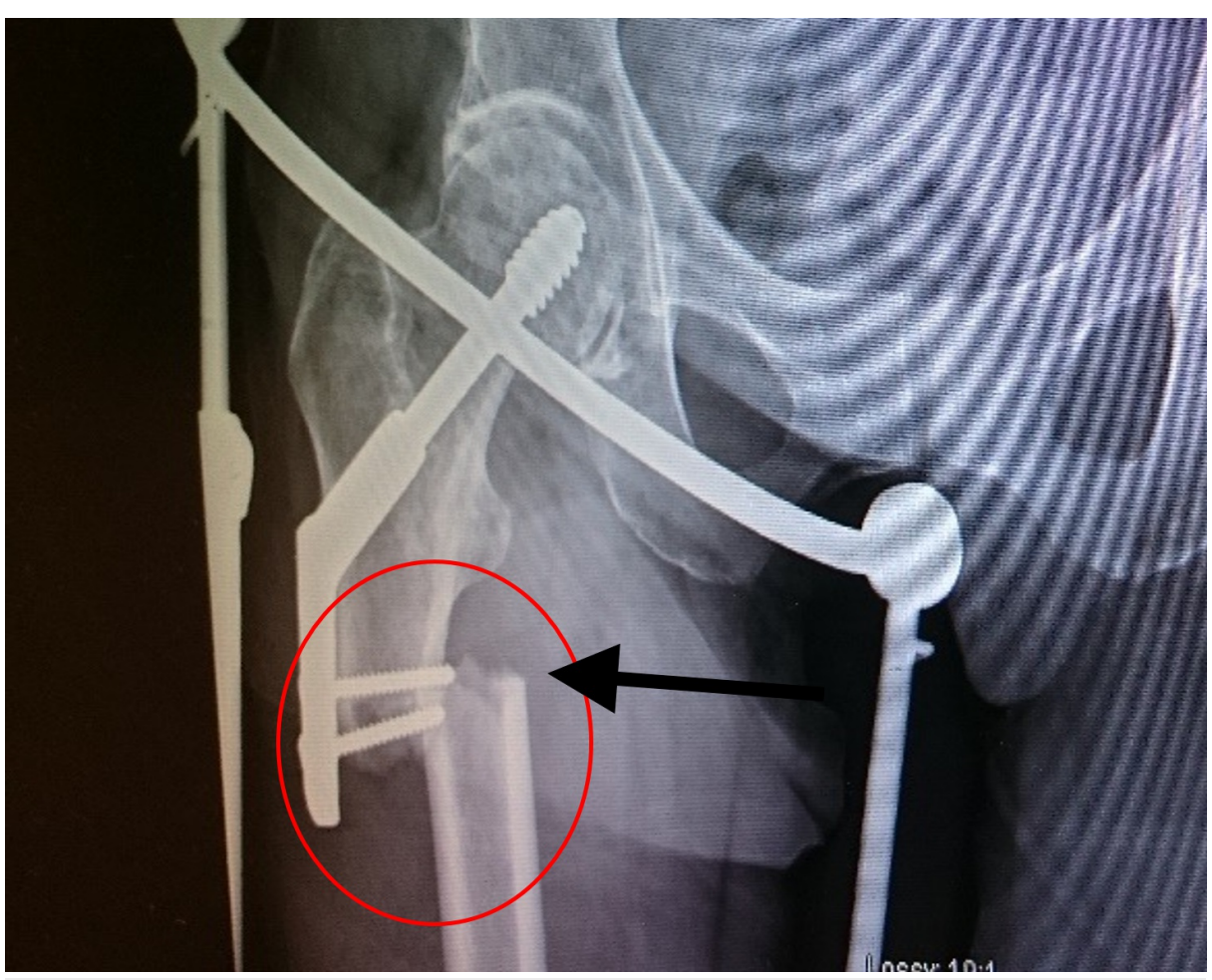

FIGURE 2: Subtrochanteric femur fracture 


\section{Cureus}

She was started on alendronate therapy in 2011 for osteoporosis. She had a fracture in the left distal radius in 2013 and underwent manipulation under anesthesia and Kirschner wire fixation. She experienced lowenergy trauma in January 2015 and presented with right thigh pain and inability to bear weight.

Radiological evaluation of the right femur and pelvis showed an atypical subtrochanteric femur fracture of the right leg (Figure 3). She underwent a surgical operation the next day, and a two-hole plate was removed without removing the lag screw and replaced using a DHS with an eight-hole plate (Figure 4). She started physiotherapy, and within few weeks, she was walking with a walking frame. She received regular follow-up radiological examinations.

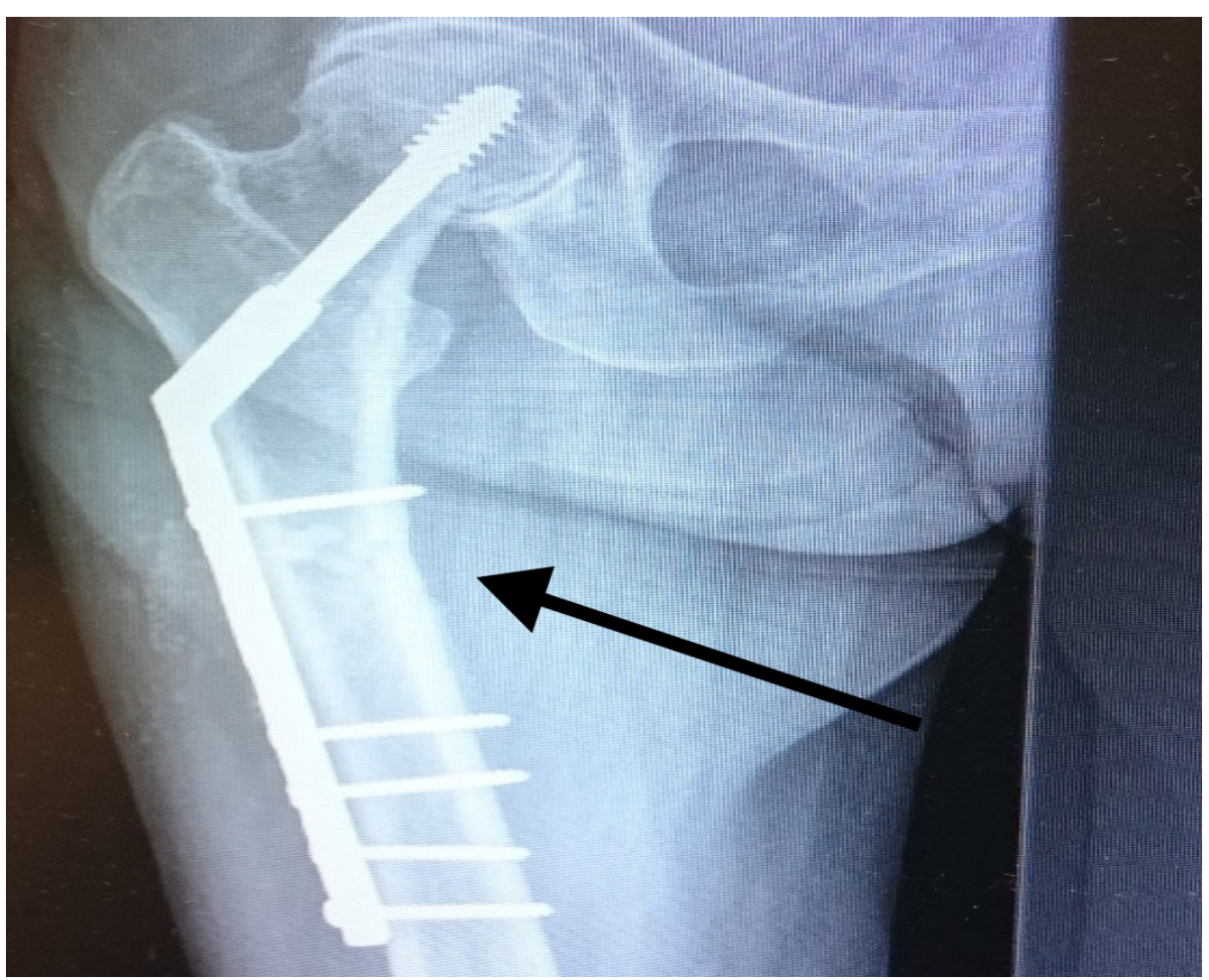

FIGURE 3: Conversion to the dynamic hip screw with a 10-hole plate

Arrow showing fracture fixation with a 10-hole dynamic hip screw (DHS) plate 


\section{Cureus}

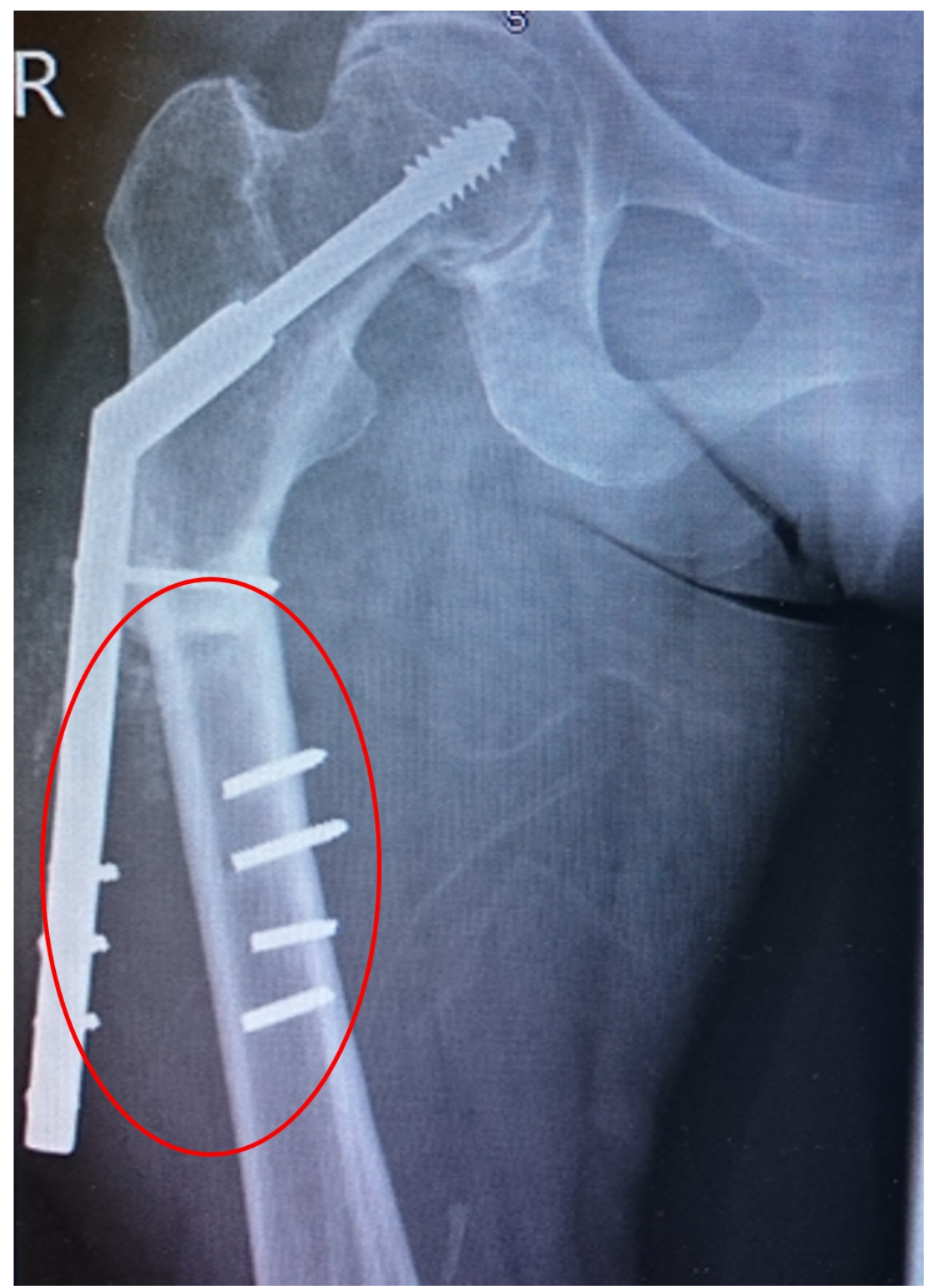

FIGURE 4: Periprosthetic fracture

Red circle denoting implant failure

Her alendronate was discontinued, and she was started on teriparatide injections. Teriparatide is a synthetic parathyroid hormone that exists naturally in the body. She was undergoing this treatment when, in April 2015, she had another low-energy fall and was brought to the hospital with pain in the right thigh and an inability to bear weight. On radiologic examination, she had DHS implant failure and nonunion of the subtrochanteric fracture (Figure 5). She underwent an additional surgical procedure, and the DHS was removed. A long cephalomedullary nail was inserted (Figure 6). She started physiotherapy again. The patient is receiving regular outpatient follow-up and radiologic examinations. She is walking with a walking frame and continues to receive teriparatide injections. 


\section{Cureus}

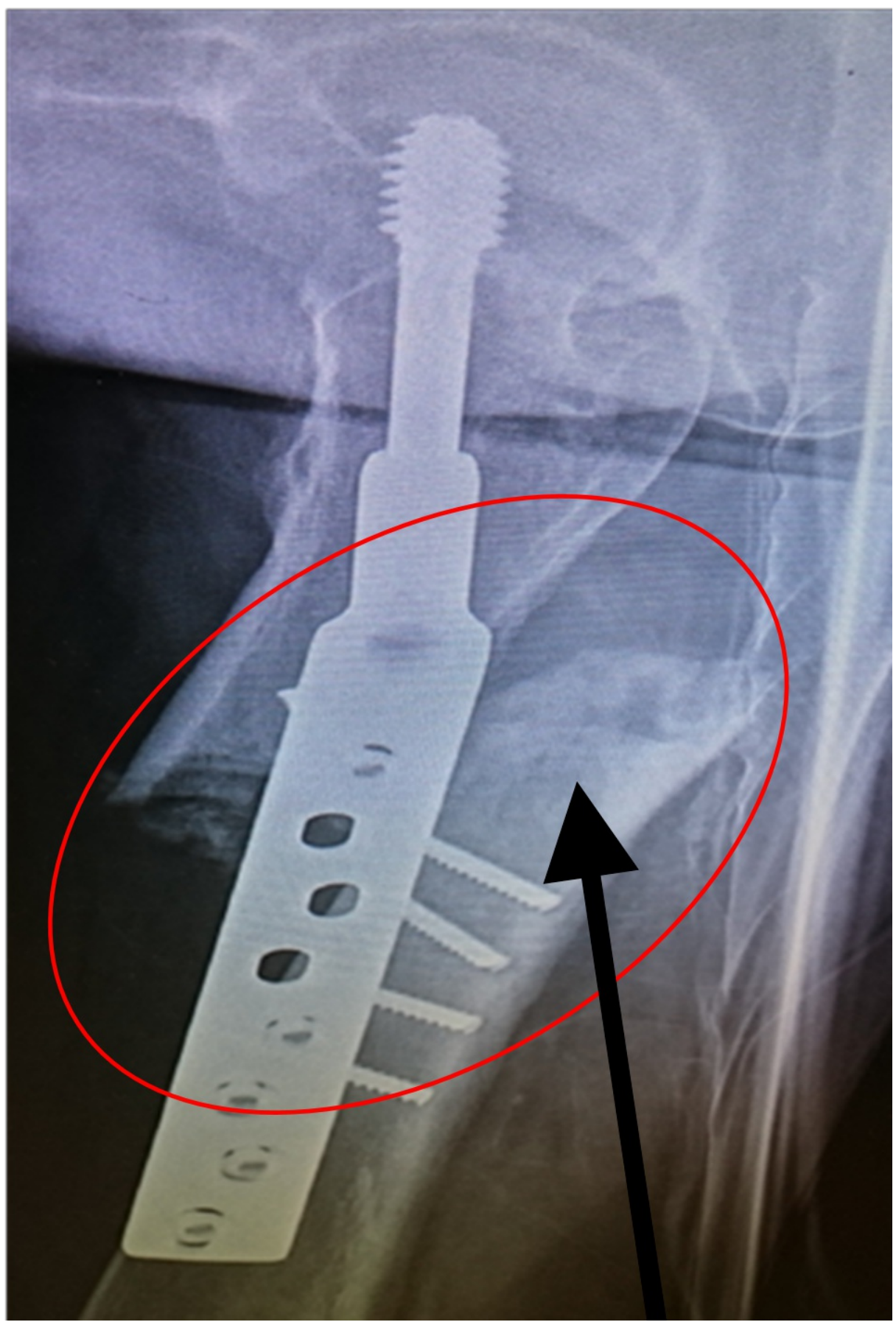

FIGURE 5: Lateral view of the periprosthetic fracture

Red circle and arrow showing the displaced periprosthetic fracture 


\section{Cureus}

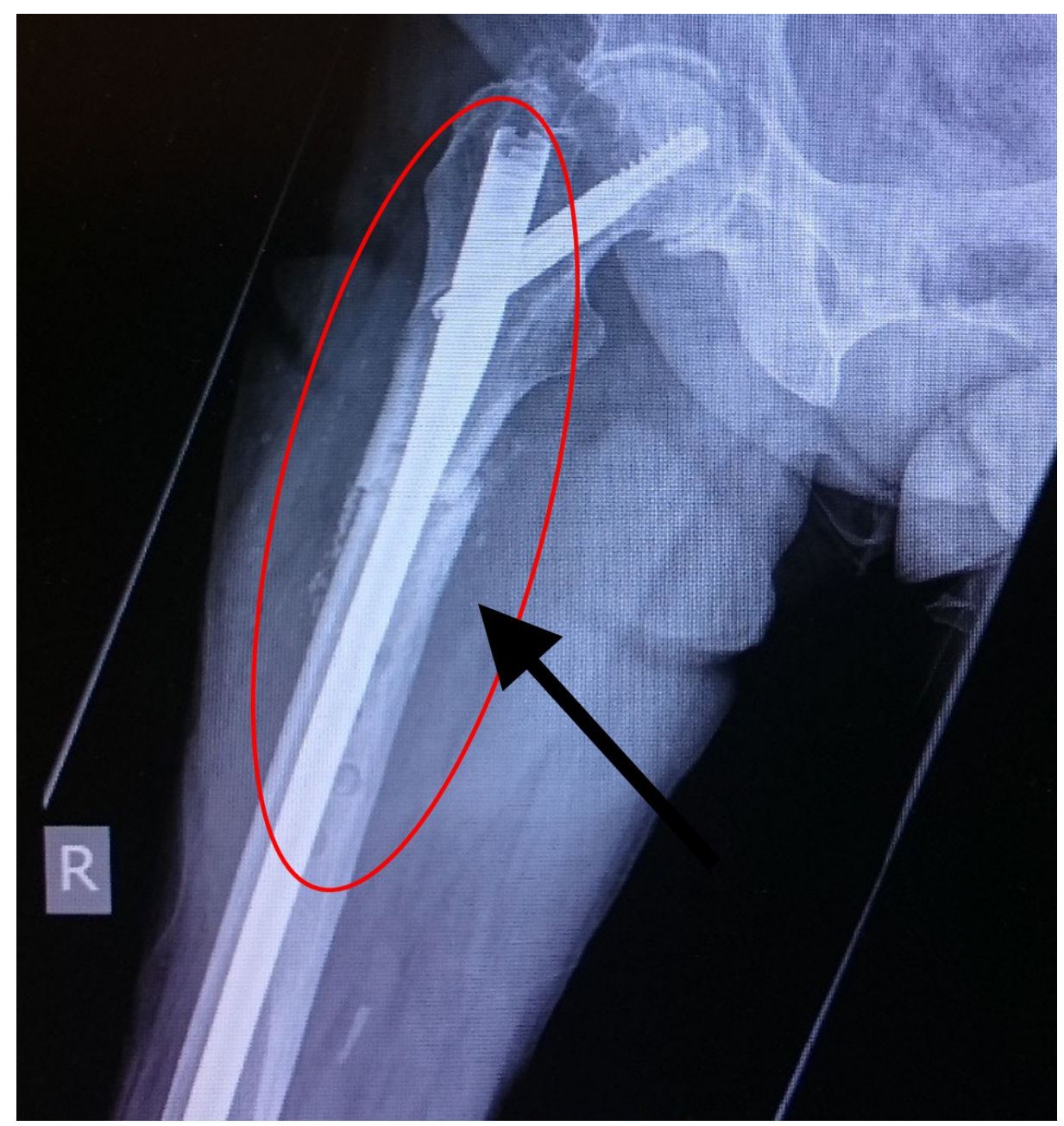

FIGURE 6: Cephalomedullary nailing of the fracture

Red circle and arrow showing the intra-medullary nailing

\section{Discussion}

Bisphosphonates are the primary pharmacological agents used to treat osteoclast-mediated bone loss due to osteoporosis, Paget's disease of bone, malignancies that metastasize to bone, multiple myeloma, and hypercalcemia of malignancy. Apart from the aforementioned uses, it is also used for low bone density and osteogenesis imperfecta as well [3-4].

Since their introduction into clinical practice, bisphosphonates have transformed the clinical care of an array of skeletal disorders characterized by excessive osteoclast-mediated bone resorption. Bisphosphates act as strong inhibitors of bone resorption by suppressing the activity of osteoclasts, and although this improves osteoporotic conditions, it reduces overall bone turnover [5]. Prolonged bisphosphonate-induced impairment of bone remodeling may cause an accumulation of micro-fractures and weakening of bone [6-7].

Atypical femur fractures are associated with the duration of bisphosphonate therapy [8]. The risk of atypical femur fracture had previously been associated with the use of glucocorticoids and proton-pump inhibitors [8]. It has been suggested in the literature that the use of bisphosphonates should be evaluated after a certain period of time [6]. Bisphosphonates are very effective for the first few years in patients with osteoporosis by preventing osteoporotic fragility fractures [9-10]. Consideration should be given to stopping bisphosphonate therapy, at least temporarily, in those patients who are assessed to be at low or low-tomoderate risk, defined as: (1) no incident fractures, (2) T-score > -2.0, and (3) no other major risk factors after a 3- to 5-year therapeutic period [8].

\section{Conclusions}

The use of bisphosphonates is beneficial for osteoporosis, but after a certain period, it can increase the risk of atypical femoral shaft fractures. Therefore, it is important for both clinicians and patients to use bisphosphonates for the correct indications but to bear in mind the duration of its use as well. 


\section{Additional Information}

\section{Disclosures}

Human subjects: Consent was obtained by all participants in this study. Conflicts of interest: In compliance with the ICMJE uniform disclosure form, all authors declare the following: Payment/services info: All authors have declared that no financial support was received from any organization for the submitted work. Financial relationships: All authors have declared that they have no financial relationships at present or within the previous three years with any organizations that might have an interest in the submitted work. Other relationships: All authors have declared that there are no other relationships or activities that could appear to have influenced the submitted work.

\section{References}

1. Neviaser AS, Lane JM, Lenart BA, Edobor-Osula F, Lorich DG: Low-energy femoral shaft fractures associated with alendronate use. J Orthop Trauma. 2008, 22:346-350. 10.1097/BOT.0b013e318172841c

2. Chua WM, Nandi N, Masud T: Pharmacological treatments for osteoporosis in very elderly people . Ther Adv Chronic Dis. 2011, 2:279-286. doi:10.1177/2040622311409972

3. Drake MT, Clarke BL, Khosla S: Bisphosphonates: mechanism of action and role in clinical practice . Mayo Clin Proc. 2008, 83:1032-1045. 10.4065/83.9.1032

4. Odvina CV, Zerwekh JE, Rao DS, Maalouf N, Gottschalk FA, Pak CY: Severely suppressed bone turnover: a potential complication of alendronate therapy. J Clin Endocrinol Metab. 2005, 90:1294-1301. 10.1210/jc.2004-0952

5. Allen MR, Burr DB: Three years of alendronate treatment results in similar levels of vertebral microdamage as after one year of treatment. J Bone Miner Res. 2007, 22:1759-1765. 10.1359/jbmr.070720

6. Black DM, Schwartz AV, Ensrud KE, et al.: Effects of continuing or stopping alendronate after 5 years of treatment: the fracture intervention trial long-term extension (FLEX): a randomized trial. JAMA. 2006, 296:2927-2938. 10.1001/jama.296.24.2927

7. Baig M: A review of epidemiological distribution of different types of fractures in paediatric age . Cureus. 2017, 9:1624. 10.7759/cureus.1624

8. Schilcher J, Koeppen V, Aspenberg P, Michaëlsson K: Risk of atypical femoral fracture during and after bisphosphonate use: full report of a nationwide study. Acta Orthopaedica. 2015, 86:100-107. 10.3109/17453674.2015.1004149

9. Girgis CM, Seibel MJ: Atypical femur fractures: a review of the evidence and its implication to clinical practice. Ther Adv Musculoskeleta Dis. 2011, 3:301-314. 10.1177/1759720X11416270

10. Baig MN, Yousaf I, Galbraith JG, Din R: Absorbable polydioxanone (PDS) suture provides fewer wound complications than polyester (ethibond) suture in acute Tendo-Achilles rupture repair. Ir Med J. 2017, $10: 566$. 